\title{
Educación superior en Costa Rica: ¿“Rankización” y descolonización?
}

\author{
Higher Education in Costa Rica: Ranking and decolonization?
}

\author{
Maurizia D' Antoni' \\ Docencia Universitaria \\ Universidad de Costa Rica \\ San José, Costa Rica \\ maurizia.dantoni@gmail.com
}

Recibido 09 de abril de 2013 • Corregido 07 de julio de 2013 • Aceptado 31 de julio de 2013

\begin{abstract}
Resumen. En este ensayo me propongo oponer la propuesta descolonizadora del ser y del conocimiento con el imaginario en la universidad pública y en las "políticas de garantía de calidad", sus propósitos y relación con el modo de producción. Planteo que desarrollar desconfianza hacia todas las modalidades colonizadoras en el conocimiento, por sí mismo "mejora la calidad" de la educación superior. Las principales conclusiones se refieren a la necesidad de discutir los riesgos de los procesos de "mejora" y de equiparación en la educación superior, indicando como relacionada y pertinente una tarea de desprendimiento epistemológico en clave descolonizadora de la universidad pública en Costa Rica.
\end{abstract}

Palabras claves. Descolonización en la educación superior, políticas de garantía de calidad, desprendimiento epistemológico, retos de la universidad pública.

Abstract. This essay contrasts the decolonization proposal of the being and of knowledge with the imaginary in the public university and in "the quality assurance policies", its purposes, and relation to production methods. The author states that developing distrust towards all forms of colonization of knowledge represent a "quality improvement" in higher education. The main findings refer to the need of discussing the risks within the "improvement" and homogenization processes in higher education having, as a relevant and inherent aspect, an epistemological detachment task as a decolonization key from the public university in Costa Rica.

Keywords. Decolonization in higher education, quality assurance policies, epistemological detachment, challenges of public university.

1 Doctora en comunicación social y especialista en psicología educativa. Es docente e investigadora en la Universidad de Costa Rica y en la Universidad Nacional. Investiga temáticas relativa a la pedagogía crítica, la psicología histórico cultural y sus aplicaciones en América Latina. Está involucrada en trabajos de acción social con población en condición de exclusión social. 
URL: http://www.una.ac.cr/educare

CORREO: educare@una.cr

\section{Descolonización y educación superior}

El momento histórico actual, caracterizado por la importancia de los medios de comunicación y el neoliberalismo en el ámbito económico, sufre el embelesamiento de la modernidad, y muchos se presentan como abanderados de la democracia y de los derechos humanos, a la vez que estas últimas promesas a menudo no se cumplen.

El presente escrito tiene la intención de señalar los riesgos de las concepciones sobre "mejoramiento de la calidad" y por ende los procesos de equiparación en la educación superior, indicando como relacionada y pertinente una tarea de desprendimiento epistemológico en clave descolonizadora de la universidad pública en Costa Rica. El culto a la mercantilización en la sociedad y en la universidad (Meoño, 2012), en efecto, se vuelve más inquietante en un proceso de reproducción constante de la colonialidad en la academia.

Hablo en el título de rankización, haciendo referencia a las políticas de "mejoramiento de la calidad" (entrecomillado porque evidentemente no las considero tales) en la educación superior. En el texto examinaré la tendencia justamente como una tendencia con resonancias políticas, y no como una propuesta teórica

Me propongo situar como encontrados la tarea que tenemos para descolonizar la mente y el imaginario en la universidad (Mignolo, 2010), y las "políticas de garantía de calidad", sus propósitos (¿ocultos?) y su vinculaciones con el modo de producción actual.

En antítesis a las citadas políticas, creo que una "mejora la calidad" de la educación superior se obtiene fomentando la duda hacia todas las modalidades colonizadoras en el conocimiento. La intención es la de partir de este escenario para poner al descubierto los riesgos que conlleva una adhesión acrítica a los procesos para el "mejoramiento de la calidad" en la educación superior, indicando como alternativa el desprendimiento epistemológico en clave descolonizadora de la educación superior.

\section{Cultura, legado, interculturalidad}

Samanamud (2012), en el contexto boliviano, reflexiona sobre la necesidad de una recuperación intercultural de su legado y logra establecer una relación entre la descolonización, entendida como un descubrimiento del pensamiento propio, y la interculturalidad.

La descolonización para él está directamente ligada a lo que fue el llamado "descubrimiento" ,que implicó ese momento histórico, con la discriminación, la explotación y la exclusión. El hecho histórico fue la colonización y el dominio en ámbito político económico de las "nuevas colonias". Colonialidad se refiere, en cambio, a un proceso vasto de control hegemónico de dominación que pasa por lo social, religioso, cultural y también político y económico. Colonialidad del poder (Samanamud, 2012, se refiere a Quijano) describe los procesos de exclusión de las personas colonizadas que están vinculados con el nacimiento del capitalismo en América. 
Samanamud (2012) define también el concepto de cultura, que comprende lo simbólico, no solamente lo conceptual, e involucra los artefactos, así que: "la identidad no tendría que "justificarse" a partir de identificarse sólo con artefactos de su cultura, sino más bien en sus formas de facilitar la vida de una cultura. Esta es una dimensión "culturalista", sino "civilizatoria" (p. 70). De esa manera, la posibilidad que tiene la vida de reproducirse supone un amplio horizonte simbólico que desemboca en lo específico de una cultura. Este es el plano donde Samanamud (2012) ubica sus preguntas sobre la interculturalidad y la posibilidad de la descolonización, consciente de que ciertas formas de determinación cultural han sido colonizadas, tal es el caso de la identidad cultural. La visión de una cultura como objetivación de la identidad le quita su dimensión simbólica, que se expresa en un plano mítico, imponiendo en cambio la monoculturalidad.

El trabajo que se tiene por hacer en América Latina a través de la descolonización es un planteamiento político de construcción que trasciende lo crítico y desemboca en sostener críticamente un pensamiento "otro": como educadores y educadoras será necesario cambiar el modo de construirse, el desde dónde de la construcción de sí, entrando a una dimensión "civilizatoria" de la pedagogía, luchando contra el colonialismo con las armas de la interculturalidad.

Es aquí que, como europea que vive en América Latina, puedo ubicarme y dar voz a mis inquietudes y malestares, relativos, en este caso, a los procesos de supuesto mejoramiento de la educación superior, ya que la dimensión colonizada de la educación es conservadora, alienante $y$, al mismo tiempo, inevitablemente xenófoba cuando construye su imaginario importado y expulsa a cualquier "otro". También en el "imperio" existe una complejidad cultural, una historia de "lenguas cortadas" e identidades culturales amenazadas (Floris, 2008), una vivencia personal que me ayuda en la identificación con las luchas decoloniales latinoamericanas.

En el campo de la educación, Samanamud (2012) señala la importancia del descubrimiento del legado pre colonial, irrecuperable en cuanto tal y que, sin embargo, se puede reconstruir. La tarea va en dos direcciones y más allá de una afirmación de lo negado. Salir del colonialismo implica afirmar el legado propio, saliendo de la relación de dominación, dice el autor, en sentido civilizatorio.

De nuevo, el propósito que involucra la resistencia a las relaciones de domino del capitalismo global es un propósito en el que siento poder participar como mujer, académica, militante política y también como extranjera que vive en América Latina y siente la necesidad de problematizar el ingreso de la universidad pública en la lógica de la "calidad".

De Souza (2008) advierte acerca de la importancia de desenmascarar los mecanismos de reproducción de la colonialidad y lo señala como tarea urgente en América Latina, con el fin de desmantelar la matriz colonial de poder.

El pensamiento decolonial (o proyecto decolonial, proyecto de la modernidad/colonialidad o teoría postoccidental, nomenclaturas producto de diferentes debates), constituye una 
URL: http://www.una.ac.cr/educare

CORREO: educare@una.cr

expresión original de la teoría crítica contemporánea aplicada a América Latina y el Caribe y sus tradiciones de estudio en las ciencias sociales.

Se ha planteado que se trata de un pensamiento articulado desde América Latina, pero su propuesta no involucra solamente esta área geográfica. El proyecto decolonial se ha inspirado, de manera crítica, a otros proyectos intelectuales y políticos de América Latina y el Caribe, para plantear con ellos diálogos constructivos que abarquen otras áreas del planeta. Se trata de una original alternativa para retomar la discusión sobre el sentido de reflexionar, a partir de la especificidad histórica y política de nuestras sociedades, entre otras. Otros proyectos centrales de la experiencia intelectual latinoamericana y del Caribe están implicados, por ejemplo, los debates sobre el colonialismo, la filosofía de la liberación, la pedagogía crítica y la teoría de la dependencia.

Entre los antecesores del proyecto decolonial, Fals (1987a) habla de colonialismo intelectual, referido a América Latina, identificándolo como un peligro en el camino para construir una sociología de la liberación. Fals afirma que es necesario disminuir el servilismo en las personas que viven en países en vía de desarrollo, cuidándose, sin embargo, de caer en la xenofobia.

Es necesario darse a la tarea de buscar la razón de ser de los latinoamericanos y las latinoamericanas, una explicación de las realidades propias. En lo específico, Fals habla del estudiantado: dice que deberían de ser capaces ". . . de manejar las técnicas de los países avanzados, y al mismo tiempo tener suficiente ingeniosidad, sentido común y seriedad para diseñar sus propios instrumentos. .." (1987, p. 21a). Más allá de esta base, y no es tarea fácil, se requeriría prepararse a poner fin a la imitación, que a veces puede ser irreflexiva, "de modelos y temas incongruentes concebidos en otras partes y para situaciones diferentes" (p. 20). A estudiantado y profesorado de la educación superior recomienda crear disidencia. La idea es de modificar la ideología, y los intereses de los países avanzados (Fals, 1987a). Más allá de las nostalgias con la cual cierta academia se dedica(ba) a admirar la resistencia y la cultura de campesinos e indígenas en América Latina, es necesario conocer las raíces epistemológicas del hacer cultura en la academia latinoamericana, para descubrir ". . . otra visión del mundo, muy distinta de la transmitida por culturas opresoras ..." (Fals, 1987b, p. 158).

La afirmación de Mignolo (2010) de que las personas que no sucumben a la fascinación de la modernización y la democracia son sujetas a todo tipo de violencia logra que conectemos inmediatamente y realísticamente con imágenes, vida vivida, referencias a personas o situaciones reales. En particular, quienes trabajamos en el ámbito educativo visualizamos la expulsión del sistema educativo como medida normalizadora de las personas resistentes (D' Antoni, J. Gómez, L. Gómez y Soto, 2013).

Desde mediados de los años setenta se difunde la postura que identifica el conocimiento como otro instrumento de colonización del saber y del ser (Mignolo, 2010). Quijano (citado por Mignolo, 2010) define colonialidad como una parte invisible y constitutiva de la modernidad y vincula la colonialidad del poder en ámbitos políticos y económicos con la colonialidad del 
conocimiento. Colonialidad del ser se relaciona con temas de género, sexualidad, subjetividad y, de nuevo, conocimiento.

Efectivamente, "la colonialidad del poder está atravesada por la colonialidad del saber, la colonialidad del ser, la colonialidad del ver; la colonialidad del hacer y del pensar..." (Mignolo, 2010, p. 12), mientras que en un contexto latinoamericano es importante restituir la voz a las historias silenciadas, las identidades reprimidas, las subjetividades cortadas en el nombre de la racionalidad, de la modernidad unificadora (Mignolo, 2010).

En América Latina (como en otras "periferias" del mundo) se adopta como propia una racionalidad constituida como universal, mientras que es la de una sola etnia. Es decir, se establece como racionalidad global la de Europa Occidental, asumiéndola como principio común. La des colonización ideológica, en cambio, daría paso a una comunicación inter cultural, un encuentro con una racionalidad propia y, por ende, nueva (Mignolo, 2010).

Por lo tanto, revelar los mecanismos de reproducción de la colonialidad es nuestra tarea urgente, con el fin de derribar la matriz colonial de poder.

\section{De América, la universidad, la colonia}

No necesariamente toda la academia en América Latina posee fuerte conciencia de la necesidad de investigar la voz propia ni mira con suspicacia las regulaciones que vienen de Europa. Un artículo mexicano reciente (Tello, de Miguel y López, 2012), por ejemplo, abre su reflexión acerca de la necesidad de analizar aspectos importantes para seleccionar plataformas de formación on-line, a la luz de las características de la educación superior basadas en las ideas del Plan Bolonia:

En la actual sociedad de la información y la comunicación se hacen necesarios varios cambios que hagan posible la adaptación a las nuevas características de la sociedad. Por ello, nos encontramos en una sociedad en continuo cambio que cada vez requiere de nosotros mayores actualizaciones. En este caso, nos vamos a centrar en dos aspectos de cambio muy importantes en la sociedad actual, por un lado la nueva concepción de la educación superior basada en la concepción de la Unión Europea, y por otro lado la necesaria incorporación de la educación al mundo digital. (Tello, de Miguel y López 2012, p. 124)

En la universidad latinoamericana la presión que se recibe es hacia los procedimientos de autoevaluación, acreditación o, por decirlo de alguna forma, de "internacionalización y equiparación de criterios", asumidos como mejora de la calidad educativa. 
URL: http://www.una.ac.cr/educare

CORREO: educare@una.cr

Agrego, con una intención hacia la autoetnografía como metodología en este ensayo, mi experiencia directa: la asistencia en una Asamblea de Escuela donde se realizaron pasos y tomas de decisiones para la autoevaluación finalizada a la acreditación de carreras. Nadie se preguntó acerca de la oportunidad de acreditar, los costos o las implicaciones. En asambleas de Escuelas anteriores, fui la única voz representando la suspicacia hacia estos procesos y mi intervención no mereció debate.

En cambio, se trata de procesos que más bien han preocupado y han sido trabajados como un riesgo para la autonomía universitaria en América Latina (Meoño, 2012). En efecto, estaríamos en una coyuntura donde emerge la escasa comprensión del momento histórico: la presencia de desigualdades insoportables se vincula con la creciente complejidad, llevando a un estado de incertidumbre generalizada. Dicho panorama pone en peligro la autonomía universitaria (Meoño, 2012), en un cuadro de mercantilización de las universidades públicas. Es necesario defender la autonomía universitaria también a través del análisis de las estrategias en curso en las universidades. La alternativa sería ir hacia la alteración de la función asignada históricamente a la universidad pública en Costa Rica, pluralista y financiada por el estado, con el fin de preparar a académicos que sean la conciencia crítica de la nación (Meoño, 2012).

De Souza (2008) escribe estar de acuerdo con una sociedad con mercado, pero no con una de mercado. Durante el año 2012 hemos visto, más claramente que nunca, que el mercado no es una entidad autónoma y sabia, capaz de autorregularse e indicar caminos para el futuro. Las políticas de globalización neoliberal que imponen el mandato de reestructurar la sociedad al servicio del mercado global (con regulación mínima) se vuelven más inquietantes en un contexto de reproducción constante de la colonialidad, cual podría ser el de la universidad latinoamericana.

En la academia, en oposición al modelo que pregona la "calidad", que se regocija de la ubicación en lo alto de los ranking, se propone trabajar para descolonizar la mente, el imaginario, los conocimientos y el ser (Mignolo, 2010).

En América latina tenemos, muy claramente definidas, las "políticas de garantía de calidad", con su "para qué" o "para quiénes", completamente coherentes, en su vinculaciones con el modo de producción capitalista, con las políticas de "apertura" de la universidad pública como vendedora de servicios (Meoño, 2012). En oposición, se encuentra la propuesta de alimentar la suspicacia hacia todas las modalidades colonizadoras en el conocimiento, operación que, por sí misma, "mejora la calidad" de la educación superior.

\section{Equiparación de criterios en la educación superior}

La educación superior ha sufrido unos cambios en términos de internacionalización y de equiparación de criterios a partir y alrededor del Pacto de Bolonia de 1999, que son motivo de 
reflexión y de preocupación en el momento actual. La preocupación reside en la dirección a la que estos cambios obligan a la universidad latinoamericana (Meoño, 2012).

El Tratado de Bolonia (De Garay, 2008) nos refiere a un proceso y luego a un documento (1999) que buscaba adaptación de todas las universidades al Ilamado Espacio Europeo de Educación Superior. En un inicio, la iniciativa podía ser considerada un experimento para la equiparación de estándares, con la anexa buena intención de allanar el camino a las personas migrantes o becarias que necesitaran reconocimiento de sus títulos de estudio en un país extranjero, en tiempo de grandes movimientos migratorios.

El tiempo ha demostrado que actividades de este tipo implican, ante todo, una dirección inquietante: la de contribuir al cambio de la universidad pública en América Latina, llevándola hacia una intención de "apertura" hacia el mercado, lo cual no era la vocación primaria de la universidad pública latinoamericana ni, seguramente, la de la Universidad de Costa Rica (Meoño, 2012).

Meoño (2012) plantea que el momento histórico complejo que vivimos, donde el capitalismo es el modo de producción imperante, ha sabido adecuarse a las complejas circunstancias de nuestra existencia actual. El sistema capitalista se ha hecho más feroz, organizándose a la vez, transformándose de manera tan efectiva que nuestra sociedad se ha vuelto un gran mercado, donde todo parece ser susceptible de convertirse en mercancía, así el sistema educativo como los recursos humanos (el agua, el aire); los mismos cuerpos humanos se compran o se roban y se venden. En un clima de mercantilización, conducido a sus extremos límites, nace una pregunta sobre educación: ¿esta debería ser concebida como un servicio o como un bien público? La pregunta es delicada en cuanto en Costa Rica la educación se ve todavía como una pieza fundamental para la transformación de la realidad y la emancipación de las personas.

Lo que se teme es que en Costa Rica (Meoño, 2012) la universidad pública vaya sucumbiendo a las presiones hacia la mercantilización, desdibujándose consecuentemente su vocación primaria: la de ser una institución pública, gratuita, capaz de proporcionarles a las personas estudiantes una formación integral, no solo técnica, que al final de la formación pueda proveer, a la sociedad de la cual forma parte, profesionales conscientes de su función en el crecimiento de la comunidad.

Los grandes informes estadísticos sobre educación nacen como fenómenos consecuentes de los acuerdos internacionales sobre equiparación de currículos y títulos de estudio: en Costa Rica tenemos el Estado de la Nación o el Estado de la Educación Costarricense. Estos documentos se encargan de funcionar, entre otros, como cajas de resonancia hacia la opinión pública, del estado de "crisis" de la educación, direccionado en clave oficialista los "datos numéricos" ( $D$ ' Antoni, J. Gómez, L. Gómez y Soto, 2013).

Unterhalter y Carpentier (2010) se hacen una pregunta pertinente acerca de la educación superior y de la justicia, relativa a cuáles intereses estamos sirviendo cuando realizamos gestiones para formar personas en la universidad. Los autores sostienen que la educación superior tiene 
URL: http://www.una.ac.cr/educare

CORREO: educare@una.cr

la potencialidad de eliminar inequidades o aumentarlas, dependiendo de sus políticas. Una educación superior globalizada serviría intereses específico y nuestra tarea como académicos y académicas es la de analizar cuáles son esos intereses. Unterhalter y Carpentier (2010) afirman que esta tarea toma la forma de un cuatralema (el lugar de un di - lema) que vincula cuatro dimensiones: crecimiento, equidad, democracia y sustentabilidad. El problema es que en este cuatralema más de una persona señalaría contradicción entre los elementos.

Es justamente ese cuestionamiento sobre qué intereses estamos sirviendo, en el contexto de una transformación de la universidad de servicio público a prestadora de servicios, la clave que podría vincular ese tema con ámbitos curriculares, como lo es la apuesta de asumir el modelo europeo que pretende mejorar o modernizar la educación superior. La transformación del modelo de educación superior, en cuanto a venta de servicios y cambios en lo curricular y metodológico dentro de la universidad, responde a una misma lógica que describo como mercantil: a esa lógica y sus peligros se opone la apuesta hacia la toma de conciencia de la especificidad epistémica de América Latina y la descolonizadora de la educación superior.

\section{¿De dónde viene Bolonia?}

El proceso consiguiente al Pacto de Bolonia, el punto de giro político que llevó a la cultura del "mejoramiento de la calidad", no surge de la nada. Como señalan Unterhalter y Carpentier (2010) y Meoño (2012), existen intereses que mueven los grandes acuerdos internacionales, las rankizaciones, las equiparaciones y nivelaciones de criterios en la educación superior. Rige estas actividades una idea de lo que es la educación superior, de su ubicación en el mercado y en el modo de producción actual, una intención sobre qué material humano debe producir. Un material humano que tiene que ser capacitado (técnicamente) e intercambiable, si es cierto que se busca abatir las fronteras con el propósito, entre otros, de permitir que los "manager" y las "manager" viajen a través de ellas.

Todos los países llegan a los procesos, a los espacios de educación superior acreditados por un recorrido que atraviesa condiciones históricas, sociales y políticas que rigen y le dan significado a los procesos educativos.

Considero interesante seguir brevemente el camino que ha llevado España al encuentro con los procesos en objeto, por ser emblemático de una situación y a la vez porque España ha influido e influye considerablemente sobre América central, entre otros, en el ámbito académico, al que se hará referencia en la segunda parte de este escrito.

En su reflexión acerca de las políticas y las problemáticas acerca de la formación del profesorado en España, Costa (2009) evidencia cómo la tendencia que llama tecnocrática se vislumbraba en España a partir de los años setentas.

Esa tendencia enfatizaba temáticas como la programación; las taxonomía de los objetivo de la educación y del rendimiento escolar; la enseñanza programada, de inspiración conductista, 
y automatizada. En ese momento histórico, en pleno franquismo, España iba preparando su Ley General de Educación, que se promulgó en 1970 bajo el ala protectora de los organismos internacionales, y apoyada por una campaña de divulgación hacia el resto del mundo.

La nueva ley nacía inspirada por los criterios del reformismo tecnocrático, auspiciado en aquellos momentos por la OCDE y el Banco Mundial, teniendo como fundamental [marco teórico] de relieve internacional y miembro del a Opus Dei Ricardo Díaz Hochleimer. . . . En los años del 1969 a 1972 se habla de modo constante de la reforma educativa y del modelo español de desarrollo educativo, con el acompañamiento del Comité de Cooperación Internacional para la reforma de la educación española, sostenido por la UNESCO. (Costa, 2009, p. 218)

La dimensión científico-técnica que en los años setentas empieza a imponerse y a sobrevalorarse (Costa, 2009) aparentemente se resiste a la explosión contemporánea de propuestas más críticas, a los desarrollos de la pedagogía crítica ( $D^{\prime}$ Antoni, en prensa) y en la misma España se opone a la tradición educativa laica y liberal de la República y a las experiencias de los años cincuentas en Cataluña, donde algunos profesores experimentaban con una perspectiva que hoy coincidiría con el modelo crítico-reflexivo (Costa, 2009).

Esa dimensión tecnocrática de la educación en España es puesta en jaque por los eventos históricos, entre ellos, y central, la muerte de Franco y el consiguiente debilitamiento del franquismo, y el proceso hacia la construcción de un estado democrático, que empezó a tener visiones y necesidades nuevas acerca de la educación. Sin embargo, los intereses en mantener un proyecto educativo ligado a la técnica eran sostenidos por el modelo de desarrollo capitalista más que por el franquismo en sí. Tanto es así que, al "explotar" de la democracia en España, a la par de la experimentación en el campo de la renovación pedagógica, con las nuevas revistas, los congresos, el interés hacia la formación del profesorado, nutridos por un espíritu que Costa (2009) define como crítico y utópico a la vez, se mantuvieron presentes tendencias educativas de otro tipo.

En los años noventa, en efecto, con la influencia del pensamiento neo-liberal y de la incipiente globalización, se producen derivas burocrático administrativas que van engullendo, en España, las vanguardias pedagógicas, convirtiéndolas en actuales temas como los "indicadores de calidad", con la obligación inherente, para el sistema educativo, a regirse por cimerios academicistas, de pericia solamente técnica.

Costa (2009) opone dos puntos de vista acerca del profesorado: la visión tecnicista promueve al profesor eficaz, un concepto sostenido por principios educativo inspirados en la economía tayleriana; mientras que la visión crítico-utópica promueve la docencia reflexiva que, en cambio, hace referencia, entre otros, al pensamiento de Dewey. 
URL: http://www.una.ac.cr/educare

CORREO: educare@una.cr

Vinculando los procesos resumidos anteriormente al tema específico de la educación superior, procede referirse ahora al Pacto de Bolonia, nacido en 1999 en Europa como momento aglutinador de concepciones educativas ligadas justamente a temas como el de la productividad, de la eficacia, del "buen uso de la técnica".

Cascante (2009) propone realizar un análisis político de los discursos que se generan alrededor del Ilamado Proceso de Bolonia. El pacto de Bolonia de 1999 funda un Espacio Europeo de Educación Superior y todo este proceso impacta fuertemente y globalmente el pensamiento y las prácticas en el campo de la educación superior. Para Cascante, son las categorías básicas que articulan el pensamiento neoliberal que llevan a la creación de espacios normativos y tecnicistas en el ámbito de la educación universitaria.

Correspondiendo a la organización pos-fordista del trabajo, el sujeto educacional neoliberal es concebido como adquisitivo, competitivo, flexible, adaptable, mutable. Este sujeto preexistente neoliberal encuentra su realización en el esfuerzo competitivo, en la posesión de bienes materiales como justa recompensa a su esfuerzo y a las competencias adquiridas en su educación. La igualdad material se convierte, más allá de la igualdad de oportunidades iniciales, en el principal obstáculo para el desarrollo humano. Un desarrollo regido también por pautas universales que se corresponden con la concepción previamente establecida por todos los seres humanos. (Cascante, 2009, p. 135)

La organización social, por lo tanto, debería responder a esas características de los sujetos y de esa manera se "explica" el fracaso de proyectos de organización social que no respondían a la "naturaleza" universal de los individuos, donde el mercado es la expresión de la naturaleza humana. Por supuestos que, según esta visión, son censurables, las iniciativas de los estados intervienen para paliar las desigualdades sociales.

En este contexto, el tema de la financiación del sistema educativo o la función de la educación superior se entienden solo como una respuesta que el Estado debería darle a las demandas de sus "clientes". De esa manera, la distribución de los recursos por parte del Estado debe hacerse según la demanda que se consiga de los clientes (empresas, estudiantes), mediante la presentación de ofertas en el mercado educativo. Quienes no cumplan con lo especificado en los programas o con el estándar de calidad establecido, perderán "clientes" y financiamiento a la vez: una buena manera de optimizar, a través de las leyes de mercado, la oferta educativa (Shahjahan, 2012).

La citada es una visión que se niega obviamente a distinguir la diferencia entre las ofertas de la educación superior privada y la tarea que el Estado ha dado a sí mismo en cuanto a educación superior: una educación que es servicio público. 
Cascante (2009) ve como perfectamente coherente con este planteamiento la propuesta de los programas basados en competencias:

La vuelta al viejo planteamiento de los programas basados en competencias ofrece buenas posibilidades para la mercantilización de la educación. Al resultar las competencias fácilmente evaluables numéricamente, resultan funcionales para establecer comparaciones entre alumnos, los centro, e, incluso, los rendimientos educativos de comunidades autónomas y Estados. Al mismo tiempo que facilita la evaluación del rendimiento del profesorado posibilitando el establecimiento de escalafones según su nivel de competencia profesional. (p. 139)

Entre los beneficios de un programa por competencias, dentro de una visión neoliberal de la educación y de la educación superior, se facilita la extensión de la educación y la posibilidad de que se convierta en negocio, a través de la estandarización que se ha mencionado y del uso de los medios informáticos. Se alcanza, así, una posibilidad infinita de expansión de la enseñanza mercantilizada.

Algunas consecuencias, según Cascante (2009) poco estudiadas del modelo educativo neoliberal involucran, por ejemplo, la penetración de la empresa privada en la educación. La idea de una educación que prosigue a lo largo de la vida equivale a un negocio en el que todas y todos vamos a consumir mercancías indefinidamente, y que nos hace clientes permanentes. Otra alerta que Cascante (2009) nos hace es que los órganos de participación se parecen cada vez más a instrumentos de gestión empresarial y, en el caso de Costa Rica, el pensamiento vuela a las juntas escolares y a las medidas tomadas por el Ministerio de Educación sobre becas y cantidad de estudiantes activos(as) en los colegios (Programa Estado de la Nación en Desarrollo Humano Sostenible, 2011).

Entre otras consecuencias de las políticas educativas neoliberales existe el efecto de creciente culpabilización social de la educación, vista a la vez como el centro del progreso social (a nivel individual o colectivo) y responsable de los males sociales por ser carente o inadecuada: desempleo, interinazgo, falta de valores, desigualdad serían todas problemáticas que podemos achacarle a la educación con sus carencias.

La desigualdad en el sistema educativo, en cambio, aparece como un producto directo de la adopción de políticas educativas neoliberales. En Costa Rica, un tema crítico no es solo la distribución de los recursos para la educación, sino que también la diferencia en el "rendimiento", la exclusión educativa o los resultados de los exámenes de admisión a las universidades públicas por parte de estudiantado proveniente de instituciones públicas versus privadas. A la vez, destaca la diferencia en el desempeño de jóvenes provenientes de algunas instituciones públicas (colegios científicos, por ejemplo) sobre otras (Programa de la nación en desarrollo humano sostenible, 2011). 
URL: http://www.una.ac.cr/educare

CORREO: educare@una.cr

Shahjahan (2012), por su lado, avanza la propuesta de que el cuerpo docente se interese en formas de resistencia contra la educación superior neoliberal. Las prácticas y las políticas actuales nos inscriben en condiciones neoliberales en la educación superior. Sin embargo, para aumentar nuestras capacidades de comprensión de esas prácticas y de esas políticas, nos favorecerían las teorías poscoloniales de la resistencia, con el afán de movilizar una crítica de la resistencia. Shahjahan (2012) plantea que existe la necesidad de poner énfasis en la resistencia transformacional, la creación de nuevas maneras de ser, de hacer y de conocer en la educación superior, con el propósito de transformar la academia.

\section{La blancura, el género, la lengua de las "periferias"}

En varios espacios se piensa la academia con los ojos de sus "grupos minoritarios", buscando los ecos de las voces y los pensamientos negados.

En la Universidad de Arizona, en los Estados Unidos, Flores (2011) se pregunta acerca de la inserción en una universidad predominantemente "blanca" de mujeres latinoamericanas. La perspectiva teórica de este estudio es el pensamiento feminista chicano y el modelo de salud cultural comunitaria de Yosso (citado por Flores, 2011).

El estudio tiene por objeto comprender un enfoque educativo (estudios étnicos) que ha demostrado tener éxito con las estudiantes afroamericanas, llenar el vacío en la literatura de los programas de estudios étnicos en la educación superior y analizar la experiencia de género de las mujeres latinas en universidades prevalentemente "blancas".

A través de un análisis temático de entrevistas en profundidad y un grupo focal realizado con seis estudiantes latinas, la autora encuentra que los estudios méxico-americanos encarnan un proceso para reclamar y redefinirse. En la tesis se identifican y analizan cuatro temas principales: recuperar el conocimiento, el yo, el espacio (o los espacios) y la redefinición de la estudiante como mujer. Los resultados sugieren la existencia de una relación positiva entre la retención de estudiantes en la institución superior de enseñanza y la existencia de programas de estudios étnicos.

Otra experiencia de utilización de un marco de estudio decolonial aplicado a la educación superior se refiere a la Universidad China de Hong Kong (Choi, 2010) y tiene que ver con una reflexión sobre el idioma que se emplea en la enseñanza.

En la primavera de 2005, los estudiantes y ex alumnos de esa universidad realizaron una protesta contra los directivos, estos sintieron que impartir los cursos en inglés se traduciría en un aumento significativo en el número de estudiantes. El estudiantado acusó a la administración de venderse, en contra de la misión de la universidad, fundada, como se indica en sus políticas, para que principal medio de enseñanza fuera el idioma chino. La universidad, por su parte, defendió su estrategia en nombre de la "internacionalización" y la necesidad de mantenerse a la cabeza en medio de una fuerte competencia en el país y en el extranjero. 
Choi (2010) examina la controversia que aparece darse alrededor de la lengua, pero que involucra el contexto más amplio de la hegemonía del inglés y el surgimiento del capitalismo académico, dos fuerzas que están indisolublemente ligadas a las sociedades no-occidentales en una era post-colonial. El libro trata de mostrar que la controversia era, en última instancia, una lucha sobre el significado de la educación universitaria, entre los que el autor llama los instrumentistas y de los humanistas en la contienda, en la era de la globalización. Lamentablemente, es el grupo de los llamado instrumentalistas que ha ganado ventaja y los parámetros del debate sobre lenguaje se han restringido.

Meza (2012) parte en su reflexión desde el cuerpo, con el fin de confrontar el poder desde la heterarquía. La temática involucra, en su tesis, la discapacidad -a la cual se acerca a través de las teorías del subalternismo, el nuestroamericanismo, el postoccidentalismo y de la decolonialidad como marco epistemológico-. Utiliza los enfoques indicados a manera de rutas para llegar a una confrontación directa con el Modelo conceptual colombiano de la discapacidad, expresado por la Maestría en Discapacidad e Inclusión Social de la Universidad Nacional de Colombia.

Sus posiciones son nuestroamericanistas y desde la subalternidad se propone develar otros cuerpos para visibilizarlos. Estos cuerpos son interlocutores reales del cuerpo que se (re)produce desde el poder colonialista en la academia y, a través del dilema de la diferencia, llegan a poder situar a la discapacidad en un territorio de antagonismos. Aquí, los discursos del modelo se despojan del poder y se dejan provocar por una comprensión diferente de los cuerpos y su relación con los estudios en discapacidad, desde la heterarquía. La decolonialidad y la no-metodología son rutas de esta investigación (Meza, 2012), con la intención de romper el camino de la investigación metodológica tradicional, que objetiviza el conocimiento, mientras que el conocimiento situado lleva a otros tipos de confrontaciones, cuerpo a cuerpo.

Rivera y Sepúlveda (2011), en su reflexión sobre la universidad y los indígenas mapuches de Chile, para la implementación de un proceso de descolonización y apertura de las estructuras académicas formales, hacia la construcción de una universidad mapuche, se tropiezan con la evidencia de que construir conocimiento no es un acto neutral.

Al contrario, se trata de un proceso vinculado con la subjetividad, que pone en primer plano la pertenencia a una realidad sociocultural de quienes producen saberes. Los ámbitos socio culturales, a su vez, son determinantes en la aproximación a distintos procesos y contextos sociopolíticos, así que la construcción de conocimiento es una actividad ligada, en su esencia, a pautas culturales definidas. Lo anterior comporta la necesidad, por parte de los actores y actrices del proceso educativo, de tomar en cuenta el sello identitario de las personas implicadas.

Rodríguez (2009) trabaja las reformas en la educación superior boliviana, una sociedad multicultural, con una óptica descolonizadora. El mestizaje es visto en Bolivia como un elemento unificador, producto de la revolución nacional de 1952, y a partir del sustrato de diferentes temporalidades históricas y culturales, la nación debe aprender a pensarse en clave pluralista, reemplazando el estado centralista y mono cultural existente. El actual presidente de Bolivia, Evo Morales es expresión de un movimiento indígena para el reconocimiento de pluralidad jurídica, 
URL: http://www.una.ac.cr/educare

CORREO: educare@una.cr

política, cultural. En lo educativo, se empezó en 1994 con la reforma educativa que proponía los retos de la interculturalidad, para llegar hoy a propuestas descolonizadoras que apuntan al reconocimiento de saberes, conocimientos y tecnologías indígenas.

Rodríguez (2009) manifiesta preocupación hacia lo que define la mercantilización de la educación secundaria, aunada a la desarticulación de los dispositivos de política pública, la permeabilidad a presiones, las imposiciones de elementos externos al estado boliviano para la creación de instancias reguladoras, algunas de las cuales se crearon para quedar luego sin aplicación real. Este es el caso, por ejemplo, de las Pruebas de Admisión Académica. Desde 2006 en Bolivia existe una tendencia hacia una educación universitaria indigenista, indianista u originaria, que contiene tendencias opuestas a la autonomía universitaria así como otras más interesadas en una reforma de la universidad que incorpore las demandas de los movimientos sociales.

La propuesta de Rodríguez (2009) de descolonizar la educación se opone a la citada idea de interculturalidad que contenían reformas educativas anteriores. La descolonización, en este sentido, se basaría en pensar lo educativo desde sí mismos, desde la cultura propia de los pueblos indígenas, con una des occidentalización del conocimiento, con la eliminación de prácticas de aula que imponen la ideología colonizadora y la discriminación indígena. Los enfoques indígenas han padecido 500 años de aculturación y, aún así, la apuesta es a la contextualización de los procesos de aprendizaje desde las visiones culturales indígenas.

Los procesos de descolonización de la educación superior, según Rodríguez (2009), involucran el ámbito educativo y lo sobrepasan, para implicar:

Aspectos de orden cultural, (relaciones de hegemonía cultural), con cuestiones de orden político y social (relaciones de dominación desde la variable cultura-etnia) con asuntos referidos a la estructura del ordenamiento jurídico social y territorial, y de concepción incluso el Estado, cuestiones de orden económico (pobreza); asuntos de orden subjetivo (construcción de identidades, autoestima, autoidentificación). Asimismo, intervienen cuestiones de orden estructural institucional en relación al sistema educativo y sus instituciones (lo esencial o inherente a la naturaleza de las instituciones y el contenido). (p. 523)

La síntesis ofrecida por Rodríguez (2009) de los ámbitos a tomar en cuenta para trabajar el tema de la descolonización en la educación superior ofrece pistas para la reflexión y la acción que son válidas también para Costa Rica, donde los datos acerca de educación en espacios indígenas es alarmante (Programa Estado de la Nación en Desarrollo Humanos Sostenible, 2011) y aun así se está hablando simplemente de equiparar la educación de áreas indígenas a la situación general del país, es decir que no se ha llegado a una reflexión profunda y colectiva acerca de educar desde lo propio. 
En Argentina, Bianco (2012) pone el acento en la investigación biográfico-narrativa, entendida como una perspectiva alternativa que permite el acercamiento a la realidad educativa, de manera que esta modalidad investigativa se convierte en una perspectiva descolonizadora.

El formador de formadores está en el centro del análisis, en su función de persona que recoge la narración: él o ella se encarga de la escucha en la alteridad y del manejo del informe narrativo.

El aporte de Bianco (2012) interesa de manera especial, ya que la autora utiliza la investigación narrativa como una vía para trabajar la formación docente en clave descolonizadora, en el contexto actual de la universalización (forzosa) de la educación. Bianco crea un vinculo teórico entre los términos "colonización" y "colonizado" con la investigación de corte positivista, discutiendo la prevalencia que se ha otorgado a ese enfoque investigativo en la producción científica hasta la actualidad.

La investigación biográfico-narrativa representa una escucha diferente, una mirada alternativa para conocer la realidad en su complejidad y para profundizar en los procesos de subjetivación; por esta razón, al develar la realidad cotidiana de los sujetos en la educación y de la educación, se involucra al personal formador de formadores y formadoras y nos impulsa a entender "lo que sucede, por qué sucede y a quién le sucede" (Bianco, 2012, p. 91), en un proceso de develación de las tramas de relaciones en la formación docente y de los procesos de naturalización que nos ve como cómplices. Son revelados, así, los aspectos materiales de nuestro trabajo cotidiano, como docentes: la cultura y el funcionamiento de las instituciones educativas, escenario de los procesos de formación.

Enríquez (citado por Bianco, 2012) afirma que las instituciones de formación docente se conforman por instituciones de existencia, instituciones de vida, una característica que las diferencia de otras instituciones. En efecto, la misma razón de ser de un organismo como la universidad es la formación del otro, donde el otro es eje de la actividad y su importancia no reside en los aspectos cognitivos y productivos: más bien el sujeto en formación necesita ser conocido, tomado en cuenta y tenido en cuenta, investigado, comprendido. Es necesario dedicarse al conocimiento profundo de la institución, de sus prácticas y de los sujetos que la conforman con sus respectivas biografías.

Bianco (2012) se remite a Freire cuando analiza las implicaciones que tiene el saber, no entendido como algo que se agote en su autosuficiencia, sino visto como válido para la comprensión y para la acción hacia la transformación y la mejora de la sociedad donde vivimos, a través del cambio de nosotros y nosotras mismas y para sí.

En este sentido, la investigación es descolonizadora, ya que no reduce la complejidad humana, más bien aporta dimensiones para conocerla en el contexto de la intervención pedagógica e institucional. 
URL: http://www.una.ac.cr/educare

CORREO: educare@una.cr

La incógnita es la dirección que se le da a esa intención educativa. En la academia costarricense existen esfuerzos para reconocer un género dentro de la actividad formativa y se ve la autonomía universitaria como la condición de un pueblo libre, con la institución del mes de abril como mes de la autonomía universitaria y la creación de una Comisión Institucional para la Autonomía Universitaria. Estos esfuerzos, sin embargo, no están todavía articulados entre sí, en la investigación, la docencia y la acción social.

\section{Retos para la descolonización en la academia costarricense}

En la universidad pública de Costa Rica, en la práctica docente universitaria cotidiana enfrentamos la existencia de un reto bastante manifiesto, que involucra procesos de descolonización. Desde los libros que utilizamos, a los expertos (en menor medida expertas) extranjeros que invitamos, y de cuyas visitas la universidad cubre los gastos; a las características y condiciones para el conseguimiento de maestrías o doctorados, que la universidad propone o impone, todo nuestro quehacer diario concreto está implicado en toma de decisiones sobre desde dónde construimos un legado como personas formadoras en la educación superior costarricense.

Como material para el análisis podría existir el testimonio en clave auto etnográfico del profesorado más inquieto en la Universidad de Costa Rica. En este escrito existiría la posibilidad de citar casos específicos de acciones que se realizan en el lugar donde trabajo. Escojo, por varias razones de orden político, referirme a ellas solamente a modo de preguntas o recomendaciones.

De Souza (2008) muestra cómo Europa crea la ciencia moderna para legitimar su expansión colonial. Esta nueva ciencia contiene premisas axiológicas y epistemológicas que comprenden la visión del mundo como máquina o la creencia en una realidad objetiva independiente de la percepción, da suma importancia a la traducibilidad en términos matemáticos de todo lo que es científico, o la visión de que la finalidad última de la ciencia es la de predecir, controlar y, finalmente, explotar la naturaleza que se conoce.

Son los Estado Unidos quienes, a partir de la Segunda Guerra Mundial, le venden al mundo, y a América Latina, la idea del desarrollo (De Souza, 2008) como sinónimo de globalización y de modernización.

Rasgos evidentes de esa idea de desarrollo contienen los documentos que incitan a mejorar, en este caso la universidad latinoamericana, en función de unos cambios sociales que exigirían avanzar, involucrando, en este "avance", las tecnologías para la comunicación (Mollis, 2010). La equivalencia progreso/desarrollo está en crisis con la crisis económica en Europa occidental y en los Estados Unidos; sin embargo, la época histórica del industrialismo aún tiene sus revoluciones (las mencionadas revoluciones tecnológica y económica, pero también una revolución cultural) de las cuales emergen visiones del mundo que luchan para reemplazar lo existente (De Sousa, 2008). 
El peligro que corremos en la universidad es que ante la "revolución tecnológica", los cambios que se pretenden realizar no sucedan por inercia, por el peso de la misma tecnología y del mercado que la impone, sino -como dice De Sousa (2008)- para descubrir identidad, rompiendo la colonialidad el poder y, consecuentemente, la colonialidad del saber.

En nuestra universidad, esa colonialidad del saber pasa también por las referencias bibliográficas que usamos, los expertos y expertas extranjeras que contratamos, que a su vez son elemento destacaos de modas culturales, cuya fascinación nos envuelve. Referencias, expertos de fama, modelos curriculares utilizados con conciencia crítica o sin ella: son todos aportes que podemos emplear con el mayor cuidado para adherir a políticas institucionales que remiten a derechos humanos, participación democrática, inclusión en la universidad.

Sin embargo, cuando en las unidades académicas se toman decisiones sobre temas muy concretos, por ejemplo, los planes de relevos y a cuáles países enviar al cuerpo docente para que prosiga estudios de maestrías o doctorados, existe el peligro de ubicarse en esa misma lógica superior-inferior, que De Sousa (2008) ve como otra invención para la dominación.

Mi propuesta inicia, por lo tanto, con la toma de conciencia y el conocimiento de la teoría decolonial aplicada a la educación superior.

Se nos plantea descubrir toda una epistemología resistente, una epistemología del Sur: si encontráramos sugerente esta invitación, entonces, hay innumerables ámbitos de acciones que nos corresponden. Uno de ellos consistiría en asumir la tarea de verificar, en los espacios cotidianos del quehacer docente de la educación superior, los peligros, las fascinaciones por las modas culturales foráneas, las agendas ocultas en las políticas de rankizaciòn de las universidades, equiparación de criterios, acreditación, "mejoramiento de la calidad": una serie de iniciativas que, en mi opinión, se mueven en la misma dirección.

En el ámbito educativo, Samanamud (2012) propone ampliar el debate desde los ámbitos pedagógico-técnicos que se enfocan solamente en el cambio de los currículos y darse a la tarea de la recuperación de la identidad: que se dé también una derrecreación de esa identidad.

Se trata evidentemente de un trabajo mucho mayor, que corre para atrás el punto de arranque de los procesos de calificación de la universidad pública en el surcontinente. La comparación de la académica latinoamericana, a través de ranking y con base en criterios de "calidad" establecidos con un ojo (o los dos), con el mercado no produce otra cosa que desdibujar la pregunta fundamental que informa la tarea para la reconstrucción y la recreación de una universidad pública en clave decolonial, y constituye una pregunta compleja sobre la recuperación de un legado. 
URL: http://www.una.ac.cr/educare

CORREO: educare@una.cr

\section{Referencias}

Bianco, I. L. (2012). La investigación biográfico-narrativa o el desafío de descolonizar nuestra mirada. Revista de Educación, 3(4), 89-100. Recuperado de http://fh.mdp.edu.ar/revistas/ index.php/r educ/article/view/88/151

Cascante, C. (2009) ¿Refundar Bolonia? Un análisis político de los discursos sobre el proceso de creación del espacio europeo de educación superior. Revista Española de Educación Comparada, 15, 131-161. Recuperado de http://e-spacio.uned.es/fez/eserv. php?pid=bibliuned:reec-2009-15-3060\&dsID=PDF

Choi, P. K. (abril, 2010). Weep for Chinese University: A Case Study of English Hegemony and Academic Capitalism in Higher Education in Hong Kong [Lamento por la Universidad China. Un estudio de casos de hegemonía inglesa y capitalismo académico en la educación superior en Hong Kong]. Journal of Education Policy, 25(2), 233-252. Recuperado de http:// www.tandfonline.com/doi/abs/10.1080/02680930903443886

Costa, A. (mayo-agosto, 2009). Políticas y problemática de la formación del profesorado en España (1969-2004): La revista de educación en el contexto de la agenda educativa internacional. Revista Diálogo Educacional, 9(27), 215-248. Recuperado de http://www. redalyc.org/articulo.oa?id=189117298002

D' Antoni, M. (En prensa). Preguntas sobre la escuela secundaria en contexto y un modelo pedagógico de la crisis.

D’ Antoni, M., Gómez, J., Gómez, L. y Soto, J. F. (2013). La escuela en cuestionamiento. Diálogos problematizadores sobre la resistencia escolar en procesos pedagógicos emergentes. San José, Costa Rica: Editorial Arlekín.

De Garay, A. (abril-junio, 2008). Los acuerdos de Bolonia; desafíos y respuestas por parte de los sistemas de educación superior e instituciones en Latinoamérica. Universidades, 58(37), 17-36. Recuperado de http://www.redalyc.org/articulo.oa?id=37311274003

De Souza, J. (junio, 2008). Desobediencia epistémica desde Abya Yala (América Latina). Tiempos de descolonización y reconstrucción en el pensamiento social latinoamericano. Ponencia presentada en el I Congreso Internacional Pensamiento Social Latinoamericano: Perspectivas para el siglo XXI. Cuenca, Ecuador. Recuperado de http://www.ts.ucr.ac.cr/ binarios/osadia/osadia-00002.pdf

Fals, O. (1987a). ¿Es posible una sociología de la liberación? En O. Fals (Aut.), Ciencia propia y colonialismo intelectual. Los nuevos rumbos (3a ed., pp. 15-23). Bogotá: Carlos Valencia Editores.

Fals, O. (1987b). Por un conocimiento vivencial. En O. Fals (Aut.), Ciencia propia y colonialismo intelectual. Los nuevos rumbos (3a ed., pp. 153-165). Bogotá: Carlos Valencia Editores. 
Flores, A. I. (2011). Decolonizing Minds: The Experiences of Latina Mexican American Studies Majors at a Predominately White University [Descolonizar las mentes. Las experiencias de mujeres latinoamericanas, mexicanas, en estudios americanos como material principal en una universidad prevalentemente blanca]. (Tesis de maestría). University of Texas at Austin. Recuperado de http : / /hdl .handle .net /2152 /ETD -UT -2011 -05-3564

Floris, G. (2008). La fabbrica degli ignoranti. La disfatta della scuola italiana.[La fábrica de los ignorantes. La derrota de la escuela italiana]. Milán: Rizzoli.

Meoño, R. (abril, 2012). Autonomia universitaria. Conquista en permanente disputa. Conferencia dictada en el Día de la Autonomía Universitaria. Recuperado de http://www.ucr.ac.cr/ medios/documentos/2012/Conferencia-escrita-AU-Conquista-en-permanente-disputa. pdf

Meza, E. H. (2012). Caminos otros para llegar al cuerpo nasa (Tesis de maestría). Universidad Nacional de Colombia, Facultad de Medicina, Bogotá, Colombia. Recuperado de http:// www.bdigital.unal.edu.co/6344/

Mignolo, W. (2010). Desobediencia epistémica: Retórica de la modernidad, lógica de la colonialidad y gramática de la descolonialidad. Buenos Aires: Del Signo.

Mollis, M. (2010). Las transformaciones de la educación superior en América Latina: ¿ldentidades en construcción? [Editorial]. Educación Superior y Sociedad, 15(1), 11-23. Recuperado de http://ess.iesalc.unesco.org.ve/index.php/ess/article/view/366/305

Programa Estado de la Nación en Desarrollo Humano Sostenible. (2011). Estado de la educación 3 (3a ed.). San José, Costa Rica: CONARE.

Rivera, F. y Sepúlveda, B. (2011). Hacia la descolonización del conocimiento en América Latina: Reflexiones a partir del caso mapuche en Chile. Cuadernos Interculturales, 9(17), 113-133. Recuperado de https://docs.google.com/viewer?url=http://www.redalyc.org/ pdf/552/55222591009.pdf\&chrome=true

Rodríguez, R. (2009). Debates y desafíos: Reformas de la educación superior en Bolivia, una sociedad multicultural. Policy Futures in Education, 7(5), 513-531. Recuperado de http:// www.wwwords.co.uk/pdf/validate.asp?j=pfie\&vol=7\&issue $=5 \& y e a r=2009 \&$ article $=6$ Ostria PFIE 75 web

Samanamud, J. (2012). Bolivia: Descolonización, interculturalidad y educación. ALAI América Latina en Movimiento, 36(474), 25-29. Recuperado de http://alainet.org/active/54308\&lang=es

Shahjahan, R. A. (2012). From "no" to "yes": Postcolonial Perspectives on Resistance to Neoliberal Higher Education [De "no" a "sí": Perspectivas poscoloniales sobre la resistencia a los neoliberals de educación superior]. Discourse: Studies in the Cultural Politics of Education, 1-14. doi: 10.1080/01596306.2012.745732 


\section{URL: http://www.una.ac.cr/educare}

CORREO: educare@una.cr

Unterhalter, E. y Carpentier, V. (2010). Introduction Whose Interests are We Serving? Global Inequalities and Higher Education [Introducción a qué intereses se están sirviendo? Inequidades globales y educación superior]. En E. Unterhalter y V. Carpentier (Eds.), Global Inequalities and Higher Education: Whose Interest are we Serving? [Inequidades globales y educación superior: Qué intereses están sirviendo?]. New York: Palgrave McMillian.

Tello, I., de Miguel, L. y López, M. D. (2012). Entornos personales de aprendizaje en el espacio europeo de educación superior. RIED: Revista iberoamericana de educación a distancia, 15(2), 123-142. Recuperado de http://e-spacio.uned.es/fez/eserv.php?pid=bibliuned:revistaRied2012-15-2-3270\&dsID=Documento.pdf

\section{Cómo citar este artículo en APA:}

D’ Antoni, M. (2013). Educación superior en Costa Rica: ¿"Rankización” y descolonización? Revista Electrónica Educare, 17(3), 21-40. Recuperado de http://www.revistas.una.ac.cr/index.php/EDUCARE/issue/current

Nota: Para citar este artículo en otros sistemas puede consultar el hipervínculo "Como citar el artículo" en la barra derecha de nuestro sitio web:

http://www.revistas.una.ac.cr/index.php/EDUCARE/index 